\title{
EMG Analysis of Vastus Medialis Obliquus/ Vastus Lateralis Activities in Subjects with Patellofemoral Pain Syndrome before and after a Home Exercise Program
}

\author{
ZHANG QI ${ }^{1,2)}$, G.Y.F. NG ${ }^{3)}$ \\ 1)Department of Physical Therapy, China Rehabilitation Research Center Affiliated Beijing \\ Boai Hospital: Beijing, 100068, China. \\ TEL +86 10-67563322-5101, E-mail: zhangqixbq@yahoo.com.cn \\ ${ }^{2)}$ Capital Medical University School of Rehabilitation Medicine \\ ${ }^{3)}$ Department of Rehabilitation Sciences, The Univerisity of Hong Kong Polytechnic
}

\begin{abstract}
The objective of this study was to analyze the effects of an 8-week home exercise program with and without electromyographic biofeedback on the relative activations of the vastus medialis obliquus and vastus lateralis using electromyographic measurement in patients with patellofemoral pain syndrome. Twenty-six subjects were randomly allocated into "exercise only" (Group 1) and "electromyographic biofeedback + exercise" (Group 2) groups. Both groups performed the same exercise program and Group 2 received electromyographic biofeedback from a system that provided real-time information on the relative activations of the vastus medialis obliquus and vastus lateralis during the exercises. The intensity of the knee pain was also recorded on a pain severity scale, respectively. These parameters were measured before and after the 8-week home exercise program. The results show that Group 1 had insignificant changes in the vastus medialis obliquus/vastus lateralis electromyographic ratio $(\mathrm{p}=0.355)$, whereas Group 2 had significantly a higher vastus medialis obliquus/vastus lateralis electromyographic ratio $(\mathrm{p}=0.017)$ when performing normal activities throughout a 6-hour assessment period. The present results reveal that the incorporation of electromyographic biofeedback into a home exercise program could facilitate the activation of the vastus medialis obliquus muscle, such that the muscle could be preferentially recruited during daily activities.
\end{abstract}

Key words: Patellofemoral pain syndrome, Vastus medialis obliquus, Vastus lateralis, Electromyographic biofeedback

(This article was submitted Nov. 1, 2006, and was accepted Jan. 9, 2007)

\section{INTRODUCTION}

Patellofemoral pain syndrome (PFPS) is often referred to as anterior knee pain ${ }^{1)}$ and is a common condition presenting to physiotherapists. The patient usually complains of insidious onset of vague, activity-related pain coupled with evidence of wasting of the vastus medialis obliquus muscle
$(\mathrm{VMO})^{2)}$. The normal patellofemoral joint relationship is disturbed, resulting in an uneven distribution of shearing and compressive forces acting on the patellofemoral joint during normal activity $^{3)}$.

Electromyographic (EMG) studies of normal subjects have revealed that the $\mathrm{VMO} / \mathrm{VL}$ (vastus lateralis) activity ratio is about $1: 1^{4,5)}$, whereas 
EMG recordings in patients with PFPS have shown that the ratio of $\mathrm{VMO} / \mathrm{VL}$ is less than $1: 1$. They typically exhibit a marked decrease in VMO activity compared with VL, and also decreased ability of VMO to produce medial pull on the patella to counteract the lateral pull produced by $\mathrm{VL}^{6}{ }^{6}$. Therefore, a physiotherapy exercise program for re-establishing balance between the $\mathrm{VMO} / \mathrm{VL}$ has become an integral part of rehabilitation for patients with PFPS, but the effects on VMO/VL activity ratios have been equivocal and there is no consensus on a generally accepted treatment approach.

Accordingly, this study was conducted to investigate the knee muscle activation after a home exercise program with or without the use of an EMG biofeedback system, to determine if biofeedback in combination with exercise has a role in the home exercise programs of subjects with anterior knee pain. The significance of this study was to establish the evidence base for a suitable and effective home exercise program for the rehabilitation of subjects with anterior knee pain.

\section{METHODS}

Twenty-six subjects (16 females and 10 males) diagnosed with PFPS by a registered doctor participated in this study. The inclusion criteria were males and females aged between 20-55 years with insidious onset of anterior knee pain unrelated to traumatic incident within the last 6 months, who had preferably not received physiotherapy treatment before, and who also experienced anterior knee pain in at least 2 of the following activities: ascending stairs, descending stairs, squatting, kneeling, prolonged sitting, hopping, or jumping ${ }^{7,8)}$. All subjects gave their written consent prior to participation.

\section{Experimental procedures}

A pretest-posttest, double-blinded design was used in this study. At the initial assessment, each subject's medical history, demographic data and their pain severity score were recorded. Then VMO/VL EMG activities were recorded by a surface EMG system for 6 hours during which the subject would assume a normal daily life.

The subjects were randomly assigned into 2 groups: the 'exercise only' group (Group 1) and the 'EMG biofeedback + exercise' group (Group 2) with 13 subjects in each group. Subjects of Group 2 did the exercise program with the assistance of biofeedback, whereas subjects in Group 1 engaged in the same exercise program with no biofeedback assistance. All patients performed their home exercise program under the guidance of an experienced physiotherapist. During the 8-week home exercise program, patients were regularly followed with telephone calls from the physiotherapist and they were reminded not to change their normal daily activity. No medication or other treatments were prescribed.

In addition to the relative activity of $\mathrm{VMO} / \mathrm{VL}$, the intensity of the knee pain during daily life was recorded on a pain severity scale (PSS). The PSS was developed as a visual analogue scale (VAS) and comprises 10 statements for performing various activities. The extent of pain is indicated by perpendicular lines with the descriptors of "none" and "unbearable" at either end. The score from each statement was determined by measuring the distance from the left end along the line where the subject placed their mark. These two evaluations were performed on all subjects prior to the beginning of their physiotherapy exercise program, and finally at the completion of the 8-week home exercise program.

\section{Home exercise program}

Both groups of subjects received the same home exercise program which was designed based on clinical practice and research information. A standardized information leaflet in Chinese was given to each subject, so that they had a better understanding of PFPS and the exercise activity pattern.

The training protocol included 4 components: (1) warm up, flexibility and stretching exercise for lower limb muscles; quadriceps strengthening exercise emphasizing VMO activation; (3) balance and proprioceptive training of the lower limb; and (4) pylometric and agility training involving jogging, running, jumping, hopping and agility drills. This program was an 8 -week home exercise protocol with 30-minute daily exercise sessions, 3 sets per exercise (i.e. $3 \times 10$ times), at slow to functional speed within the pain-free range.

Instruction to the subjects in the "EMG biofeedback + exercise' group was to aim at achieving selective increase of VMO activity during the above exercises, while maintaining VL constant. 


\section{Electrode placement}

The electrodes were rectangular in shape with 1.5 $\mathrm{cm}$ of inter-electrode distance and built-in preamplifiers. The skin along the fiber directions of VMO $\left(55^{\circ}\right.$ to the vertical $)$ and VL $\left(15^{\circ}\right.$ to the vertical) were shaved and cleaned with alcohol. Light abrasion was performed using skin preparation gel, followed by cleaning with alcohol again. Skin resistance was checked after preparation with an impedance meter (Rochester Electro-medical, INC., USA) and a value of less than $5 \mathrm{k} \Omega$ was considered as acceptable ${ }^{9}$ (Gilmore $\&$ Myers 1983).

For the VMO muscle, the electrodes were placed on the skin about $4 \mathrm{~cm}$ superior and $3 \mathrm{~cm}$ medial to the superior-medial border of the patella ${ }^{10,11)}$. For the VL muscle, the electrodes were placed on the skin about $10 \mathrm{~cm}$ superior and $7 \mathrm{~cm}$ lateral to the superior border of the patella ${ }^{10,11)}$. The common reference electrode was placed on the ipsi-lateral fibula head. Electrodes were affixed to the skin with hypoallergenic tape.

After applying the electrodes, the wires of the two circuits were then secured to the skin with hypoallergenic tape to minimize movement artifacts and then connected to the main unit of the EMG amplifier which had a bandwidth of 20-450 Hz. Raw EMG signals were digitized by an A/D converter with a sampling rate of $1,000 \mathrm{~Hz}$. After the EMG signals were digitized, they were processed for their root mean square values and recorded on a compact flash data storage card. None of the electrodes were removed after application until completion of recording the activity over a 6 -hour period of a normal day.

\section{Testing movement}

To allow for comparison of EMG intensity between muscles, the data were normalized to the EMG values acquired during a maximal knee extension effort. This was done by asking each subject to perform a single leg sit-to-stand with the affected leg in the first minute of the recording. During this period, the EMG unit would record the signals of both VMO and VL which were regarded as the maximum activities of these muscles for later normalization with the recording. Then the subject would continue to wear the electrodes over the following 6 hours to record the EMG activities of VMO and VL.

\section{EMG signal analysis}

The EMG trace was recorded continuously for 6 hours to obtain an objective measure of the relative activities of $\mathrm{VMO} / \mathrm{VL}$ muscles. The relative activities of VMO/VL muscles of each subject were analyzed by calculating the percentage of the activity of each muscle over its own reference value in the period of recording. The EMG signals were analyzed by comparing the activity of each muscle to the $100 \%$ reference signal. The time at which the muscle worked at higher than $20 \%$ of its maximum voluntary contraction (MVC) value was regarded as functionally meaningful and this time formed the basis for analysis. The total time for $\mathrm{VMO}$ and $\mathrm{VL}$ working at their respective $20 \% \mathrm{MVC}$ or more was therefore expressed as a ratio to denote the relative activity levels of the two muscles.

\section{Data analysis}

The two sets of data obtained in 1-minute walking and 1-minute up-down stairs were used for calculating the reliability of EMG measurement with intraclass correlation coefficients (ICC $[1,1]$ ). Means and standard deviations were calculated for the descriptive characteristics. The independent $t-$ test was used to compare the demographic results, $\mathrm{VMO} / \mathrm{VL}$ EMG activity ratio and PSS scores in each group to determine whether the two groups started the study with a similar baseline. The final data were also analyzed by the independent t-test to look for significant differences between the two groups. Then the paired t-test was used to compare the difference scores before and after the home exercise program in each group. The level of significance was chosen as 0.05 .

\section{RESULTS}

The $\operatorname{ICC}(1,1)$ ranged from 0.983 to 0.999 for the two sets of data in each variable. This indicates that the reliability of the EMG measurements was very high $^{12)}$.

The demographic data, PSS scores and VMO/VL EMG ratio of the two groups before the exercise program were evaluated. The results suggest that the groups were well matched before the exercise program.

The results of the score changes in both groups before and after a home exercise program are reported in Table 1. 
Table 1. Results of the score change in the two groups before and after the home exercise program (13 subjects in each group)

\begin{tabular}{cccc}
\hline Group & Initial & Final & $p$ \\
\hline EMG biofeedback + exercise $(\mathrm{n}=13)^{*}$ & & & \\
PSS score & $41.93 \pm 18.45$ & $35.43 \pm 22.73$ & 0.302 \\
VMO/VL EMG ratio & $0.5662 \pm 0.255$ & $0.7944 \pm 0.2956$ & $0.017^{*}$ \\
Exercise only ( $\mathrm{n}=13)$ & & & \\
PSS score & $39.76 \pm 19.84$ & $29.71 \pm 21.10$ & 0.062 \\
VMO/VL EMG ratio & $0.5943 \pm 0.3291$ & $0.6798 \pm 0.2516$ & 0.355 \\
\hline
\end{tabular}

* Statistically significant.

\section{DISCUSSION}

This study was designed to investigate the effectiveness of a home exercise program on relative EMG activities of VMO and VL. More specifically, the goals of the present study were to determine whether physiotherapy exercises could positively affect the VMO/VL contraction ratio during functional daily activities in subjects with anterior knee pain and whether the VMO/VL EMG activity ratio is differentially affected with training in the same exercise protocol with or without a biofeedback program.

\section{Interpretation of the present results}

Improvements over time in the VMO/VL EMG ratio were observed in both groups with significant difference in the 'EMG biofeedback + exercise' group only after the exercise program. This implies that EMG biofeedback may be an effective adjunct to a home exercise program for patients with PFPS to increase their VMO/VL EMG ratio.

EMG biofeedback for guiding the activation of VMO was used in the 'EMG biofeedback + exercise' group in this study. The mechanism by which EMG biofeedback may have helped to increase the VMO/VL EMG activity ratio has not been previously investigated. Two explanations may be proposed. First, the novel electronic device used might have increased the motivation of the subjects in the 'EMG biofeedback + exercise' group. Second, the muscle contraction and accuracy with which EMG biofeedback provided information to the patient during the training session might have facilitated the integration of sensory cues and motor output during training.

Both groups showed a trend of reduction of pain score over time, even though the changes were not significant. This pain reduction trend indicates the clinical effectiveness of using EMG biofeedback as an adjunct to a home exercise program. Miller et al. ${ }^{13)}$ demonstrated that as pain in the knee decreased it facilitated VMO activity. The importance of the VMO muscle activity is paramount to functional abilities.

\section{Rationale for study design and implementation}

The treatment regimen in this study was standardized and many features of clinical practice which have been shown to be effective were included. Therefore, the design of this study should adequately answer the question of whether incorporating EMG biofeedback in exercise training can positively affect the VMO/VL EMG activity ratio.

The methodology chosen for this study was based on the assumption that a home exercise program was able to change the VMO/VL EMG activity ratio during normal daily life and that an exercise program is necessary for improvement of symptoms. The relative magnitude of the $\mathrm{VMO} / \mathrm{VL}$ ratio during activities of daily living is an indication of muscle synergism of the knee. It is important for physiotherapists to understand the knee's muscle activation pattern during functional activities, because it can provide a framework within which specific pathologic processes of the knee can be determined and from which, specific recommendations can be made in the rehabilitation program.

However, methodology of the present study had not been tested before and the results indicate that patients receiving a home exercise program would have differences in VMO/VL EMG ratio before and after an 8-week program. 
The importance of the standardized home exercise program

In the present study, all subjects increased their $\mathrm{VMO} / \mathrm{VL}$ EMG activity ratio after the 8-week training program regardless of whether they used biofeedback or not. Although the improvement was not statistically significant in the 'exercise only' group, the observed trend supports the use of the present exercise program.

It was believed that each of the exercise components used in this study played an important role in promoting a successful outcome. The specific exercises that were chosen for the present study included 4 components. General quadriceps strengthening exercises emphasizing recruitment of VMO included because they are commonly used in the treatment of PF disorders. Data from previous investigations ${ }^{14,15)}$ have indicated that quadriceps exercises consistently elicit the greatest EMG activity of VMO and VL in most subjects. Various exercise protocols including the use of EMG biofeedback have been proposed to restore the impaired balance between the two muscles and help in the learning process of muscular control to provide better PF stability ${ }^{16)}$.

Patella tracking can be improved (moved more medially) by VMO strengthening in combination with iliotibial band stretching and joint mobilization ${ }^{17}$. Therefore, the warm up, flexibility and stretching exercise for the lower limb muscles were essential and integral parts of the present physiotherapy treatment program.

The remaining components of the home treatment program (proprioception training and agility training) have been less extensively investigated. Proprioception has been shown to be defective in patients with PFPS ${ }^{18)}$. The authors suggest that clinicians should incorporate proprioceptive training when designing rehabilitation regimes.

Validity of outcome measurements assessing the efficacy of a home exercise program

The VMO/VL EMG activity ratio was used to present the relative activity of these muscles during daily living. It is an indicator of altered recruitment patterns and muscle dysfunction and it was believed that this ratio could provide an index of the medial and lateral forces on the patella.

It is known that patients complaining of anterior knee pain problems have an altered VMO/VL EMG activity ratio compared to healthy subjects, and that a lowering in the $\mathrm{VMO} / \mathrm{VL}$ EMG activity ratio is a predisposing factor for the development of anterior knee pain ${ }^{19}$ ). In a rehabilitation program, an increase in magnitude of the VMO/VL EMG ratio implies an increase of medial pull on the patella. Therefore, the VMO/VL EMG activity ratio appears to be an important parameter in the consideration of rehabilitation for PFPS.

Pain is the dominant feature of PFPS. The assessment of knee pain was estimated with a PSS in this study. Laprade ${ }^{20)}$ reported that the test-retest reliability of PSS was excellent (Spearman's rho $=0.95, p<0.0001)$. This indicates that pain in patients with PFPS can be reliably assessed using the PSS.

\section{Factors affecting EMG recordings}

EMG measurements can be a useful tool for physiotherapists to obtain information about the magnitude of muscle activity ${ }^{9}$. However, there are concerns about the sensitivity of surface electrodes and their potential for contamination by cross-talk. Cross-talk is an important concern when using surface electrodes for assessment. It is a confounding variable that can never be completely eliminated in any EMG study ${ }^{21)}$. However, according to Winter et al. ${ }^{22)}$, cross-talk can be decreased to $6 \%$ or less with a spacing of $5 \mathrm{~cm}$ between electrode pairs, and to less than 3\% for 7.5 $\mathrm{cm}$ spacing on the quadriceps femoris. In the present study, there was at least $8 \mathrm{~cm}$ spacing from VMO to VL between electrode pairs; thus, the cross-talk in EMG signal should have been kept to a minimum.

Despite the precautions taken during the experimental procedure, EMG activity might still vary when it is measured repeatedly over long time frames because of changes in body fat and machine calibration. Therefore, the data were normalized before data analysis in the present study. According to Cerny ${ }^{23)}$, normalization of data can eliminate the influence of the location and size of the recording electrodes.

The reference peak EMG value for this study was obtained from the activity of rising from sitting with one leg. This normalization procedure was favored by subjects who had difficulty in developing maximal extension force because of pain and discomfort. Furthermore, this activity was regarded as functionally demanding enough on the knee muscles to reflect the maximum activity a subject 
would do in normal daily living.

\section{Reliability of the EMG measurements}

During the 6-hour testing, the electrodes were not removed once they were applied to the skin. In order to ensure that electrode placement and amplification were unchanged across evaluation, the bony landmark together with skin marks were recorded on a transparency sheet at the initial assessment which was later used to guide the accurate repositioning of the electrodes in the final testing sessions.

In the present study, the reliability measurements were taken on separate days. The high ICC values provided good justification that the reliability of the EMG measures were within acceptable limits ${ }^{12)}$.

\section{Comparison with previous findings}

Many studies have been conducted to evaluate the efficacy of physiotherapy treatment $\left.{ }^{7}, 8,24,25\right)$. Among the controlled studies, only Dursun et al. ${ }^{8)}$ investigated the effectiveness of EMG biofeedback on the balance between VMO and VL when used as an adjunct to conventional therapy in 60 patients with PFPS. The biofeedback group received EMG biofeedback training and a conventional exercise program, whereas the control group received a conventional exercise program only. They found that mean contraction values of the VM at all 3 monthly measurements and VL at the end of the first month of the biofeedback group were significantly higher than those of the control group. These results show that a combination of EMG biofeedback with a conventional exercise program resulted in better function and reduction of pain.

The results of the present study are in agreement with the findings of Dursun et al. ${ }^{8)}$. In the present study, VMO/VL EMG ratio was significantly improved over time in the 'EMG biofeedback + exercise' group only, not in the 'exercise only' group. This is an indication that the addition of biofeedback could have strengthened the training of VMO, since this muscle was recruited more frequently during normal daily activities.

The present study found that a home exercise program resulted in a trend of pain reduction in both groups, and that differences between the two groups were small and not statistically significant ( $p>0.05)$. This finding is consistent with the study of Dursun et al. ${ }^{8)}$ in which, the effects of EMG biofeedback treatment and a conventional exercise program were evaluated for 1 month or longer with patients with PFPS. The results of that study showed that there was no evidence that the pain scores of the patients receiving EMG biofeedback were better than those of patients who received exercise only.

\section{Limitations of this study}

In this study, several points of research methodology need to be addressed. The multifaceted treatment program used in this study makes it impossible to determine which component was responsible for the change in the $\mathrm{VMO} / \mathrm{VL}$ EMG ratio and pain score. Also, with only 26 subjects in this study, the lack of significant differences between groups may be attributable to the relatively small sample size.

The use of $20 \%$ of maximum EMG as a cut-off point for the 6-hour analysis was arbitrarily chosen. It was based on the assumption that this trend of activation was reached in most daily functional activities, and thus it could reveal the dynamic activity patterns of VMO and VL. However, this is an assumption which has not been proved by concrete evidence.

\section{REFERENCES}

1) Hilyard A: Recent developments in the management of patellofemoral pain: the McConnell programme. Physiotherapy, 1990, 76: 559-565.

2) Garrick JG: Anterior knee pain (chondromalacia patellae). The Physician \& Sports Medicine, 1989, 17: 75-84.

3) Sikorski JM, Peters J, Watt T: Importance of femoral rotation in chondromalacia patella as shown by serial radiography. J Bone Joint Surg, 1979, 61B: 435-442.

4) Powers CM, Landel R, Perry J: Timing and intensity of vastus muscle activity during functional activities in subjects with and without patellofemoral pain. Phys Ther, 1996, 76: 946-955.

5) Miller JP, Sedory D, Croce RV: Vastus medialis obliquus and vastus lateralis activity in patients with and without patellofemroal pain syndrome. J Sports Rehabil, 1997a, 6: 1-10.

6) Davlin CD, Holcomb WR, Guadagnoli MA, et al.: The effect of hip position and electromyographic biofeedback training on the vastus medialis obliquus: vastus lateralis ratio. J Athletic Training, 1999, 4: 342352.

7) Cowan SM, Bennell KL, Hodges PW: Therapeutic patellar taping changes the timing of vasti muscle activation in people with patellofemoral pain syndrome. Clin J Sport Med, 2002b, 12: 339-347.

8) Dursun N, Dursun E, Kilic Z: Electromyographic 
biofeedback- controlled exercise versus conservative care for patellofemoral pain syndrome. Arch Phys Med Rehabil, 2001, 82: 1692-1695.

9) Gilmore KL, Meyers JE: Using surface electromyography in physiotherapy research. Aust J Physiother, 1983, 29: 3-9.

10) Lam PL, Ng GYF: Activation of the quadriceps muscle during semisquatting with different hip and knee positions in patients with anterior knee pain. Am J Phys Med Rehabil, 2001, 80: 804-808.

11) Ng GYF: Patellar taping does not affect the onset of activities of vastus medialis obliquus and vastus lateralis before and after muscle fatigue. Am J Phys Med Rehabil, 2005, 84: 106-111.

12) Portney LC, Watkins MP: Foundations of Clinical Research. Applications and Practice, 2000. USA: Appleton and Lange.

13) Miller JP, Sedory D, Croce RV: Leg rotation and vastus medialis oblique/vastus lateralis electromyogram activity ratio during closed chain kinetic exercises prescribed for patellofemoral pain. $\mathrm{J}$ Athletic Training, 1997b, 32: 216-219.

14) Soderberg GL, Minor SD, Arnold K, et al.: Electromyographic analysis of knee exercises in healthy subjects and in patients with knee pathologies. Phys Ther, 1987, 67: 1691-1696.

15) Karst GM, Jewett PD: Electromyographic analysis of exercises proposed for differential activation of medial and lateral quadriceps femoris muscle components. Phys Ther, 1993, 73: 286-295.

16) Boucher JP, King MA, LeFebvre R, et al.: Quadriceps femoris muscle activity in patellofemoral pain syndrome. Am J Sports Med, 1992, 20: 527-532.

17) Doucette SA, Goble M: The effect of exercise on patellar tracking in lateral patellar compression syndrome. Am J Sports Med, 1992, 20: 434-440.

18) Baker V, Bennell K, Stillman B, et al.: Abnormal position sense in individuals with patellofemoral pain syndrome. J Orthop Res, 2002, 20: 208-214.

19) Ficat RP, Hungerford DS: Disorders of the Patellofemoral Joint. Baltimore: Williams \& Wilkins, 1977, p 132.

20) Laprade JA: A self-administered pain severity scale for patellofemoral pain syndrome. Clin Rehabil, 2002, 16: 780-788.

21) Ng GYF, Stokes MJ: Influence of trunk muscle activity on surface electromyographic recordings from respiratory muscles in normal subjects. Physio Theo \& Prac, 1992b, 8: 11-17.

22) Winter DA, Fuglevand AJ, Archer SE: Crosstalk in surface electromyography: theoretical and practical estimates. J Electromyogr Kinesiol, 1994, 4: 15-26.

23) Cerny K: Vastus medialis oblique/vastus lateralis muscle activity ratios for selected exercise in persons with and without patellofemoral pain syndrome. Phys Ther, 1995, 75: 672-683. 